\title{
Parathyroid Hormone Levels May Predict Nonalcoholic Steatohepatitis in Morbidly Obese Patients
}

\author{
Morteza Ghoghaei ${ }^{1}$; Foad Taghdiri ${ }^{2}$; Elias Khajeh ${ }^{2}$; Farid Azmoudeh Ardalan ${ }^{3}$; Mojtaba \\ Sedaghat ${ }^{4}$; Sepideh Hosseini Shirvani ${ }^{5}$; Shadi Zarei ${ }^{2}$; Karamollah Toolabi ${ }^{6,}$ \\ ${ }^{1}$ Department of Internal Medicine, Imam Khomeini Hospital, Tehran University of Medical Sciences, Tehran, IR Iran \\ ${ }_{3}^{2}$ Endocrinology and Metabolism Research Center, Vali-Asr Hospital, Tehran University of Medical Sciences, Tehran, IR Iran \\ 3 Department of Pathology, Imam Khomeini Hospital, Tehran University of Medical Sciences, Tehran, IR Iran \\ ${ }_{5}^{4}$ Department of Community Medicine, Tehran University of Medical Sciences, Tehran, IR Iran \\ ${ }_{5}^{5}$ School of Medicine, Babol University of Medical Sciences, Babol, IR Iran \\ 6 School of Medicine, Babol University of Medical Sciences, Babol, IR Iran
Department of General Surgery, Imam Khomeini Hospital, Tehran University of Medical Sciences, Tehran, IR Iran \\ ${ }^{*}$ Corresponding Author: Karamollah Toolabi, Department of General Surgery, Imam Khomeini Hospital, Tehran University of Medical Sciences, Tehran, IR Iran. Tel: +98-9123212787, \\ Fax:+98-66486772, E-mail: Dr.K.Toolabi@gmail.com
}

Received: May 3, 2015; Revised: June 12, 2015; Accepted: June 16, 2015

\begin{abstract}
Background: Obesity as a worldwide health problem is associated with nonalcoholic steatohepatitis (NASH). Since severe liver injury may be present in asymptomatic obese patients and a definite diagnosis of nonalcoholic steatohepatitis can only be made after an invasive procedure of liver biopsy, there is a need for noninvasive methods to predict the probability of NASH.

Objectives: To investigate the role of vitamin D endocrine system in predicting the probability of presence of NASH in asymptomatic morbidly obese candidates of bariatric surgery.

Patients and Methods: From December 09 to March 11, every patient undergoing bariatric surgery had a liver biopsy. Nonalcoholic steatohepatitis was diagnosed using the Lee's criteria, the baseline labs obtained and the association between laboratory data and presence of NASH assessed.

Results: Forty-six patients ( 34 women, aged $36.5 \pm 10.6$ years) were analyzed. The mean levels of liver enzymes were significantly higher in the group with NASH (Pvalue $<0.01$ ). In an unadjusted logistic model, PTH was the only variable in vitamin D endocrine system which was significantly associated with NASH (odds ratio (OR): 1.04, 95\%CI:1.01-1.07). After adjustment for possible confounding factors, age (OR: 1.22, 95\%CI: 1.00 - 1.50) and PTH (OR: 1.08, 95\%CI: 1.01-1.16) were predictive factors for NASH (Pvalue < 0.05 ).

Conclusions: Elevated serum PTH level was the predictive factor for NASH in morbidly obese patients. Also, we reported elevated serum liver enzymes, high serum PTH levels and older age as predictors of NASH in patients seeking obesity surgical treatments.
\end{abstract}

Keywords: Steatohepatitis; Obesity; Parathyroid Hormone; Bariatric Surgery

\section{Background}

Obesity as a major worldwide health problem is associated with a group of chronic diseases including diabetes mellitus, cancer, cardiovascular disease (1-3) and nonalcoholic fatty liver disease (NAFLD), which is a broad spectrum from steatosis, non-alcoholic steatohepatitis (NASH) through cirrhosis and even hepatocellular carcinoma (HCC) (4). Obesity is the most common cause of $\operatorname{NAFLD~and~} \operatorname{NASH}(5,6)$ and NASH is known as the leading cause of cryptogenic cirrhosis (7).

Since severe liver injury may be present in asymptomatic obese patients and a definite diagnosis of NASH can only be made after an invasive procedure of liver biopsy associated with pain, high costs, pathologist-dependency and multiple risks, there is a need for noninvasive methods to predict the probability of NASH $(8,9)$. Several risk factors such as older age (10), high body mass index (BMI)
(11), hypertension (12), insulin resistance (12), diabetes mellitus (13) or liver function tests abnormalities (14) have been considered as predictive factors for this serious liver disease.

On the other hand, many studies noted that vitamin D deficiency, increased serum parathyroid hormone (PTH) level and some other serum biomarkers are associated with obesity and some of its related comorbidities such as metabolic syndrome (15-18).

\section{Objectives}

The main aim of the present study was to investigate the possible role of vitamin $\mathrm{D}$ endocrine system in predicting the probability of presence of NASH in asymptomatic morbidly obese subjects selected for bariatric surgery in a surgery hospital in Iran.

Copyright ( 2015, Kowsar Corp. This is an open-access article distributed under the terms of the Creative Commons Attribution-NonCommercial 4.0 International License (http://creativecommons.org/licenses/by-nc/4.0/) which permits copy and redistribute the material just in noncommercial usages, provided the original work is properly cited. 


\section{Patients and Methods}

Between December 2009 and March 2011, a total of 47 consecutive morbidly obese patients presented in our bariatric surgery center (Erfan hospital, Tehran, Iran) to undergo laparoscopic bariatric surgery. According to the national health institute's guidelines, bariatric surgery criteria was defined as BMI $>40 \mathrm{~kg} / \mathrm{m}^{2}$ or BMI $>35 \mathrm{~kg} / \mathrm{m}^{2}$ with an obesity related comorbidity and failure to lose weight by nonsurgical treatments $(19,20)$. We excluded patients with previously proven hepatic diseases (hemochromatosis, Wilson's disease, etc.), patients younger than 18 years old, consumers of more than $200 \mathrm{~g}$ of alcohol per week, subjects with positive viral markers (viral hepatitis or Human Immunodeficiency Virus) or positive antinuclear antibody (ANA), impaired renal function and those who receiving medications related to fatty liver disease, such as Tamoxifen, Valproate, Amiodarone, Diltiazem, Estrogens, corticosteroids and Methotrexate, or taking drugs affecting calcium or vitamin D metabolism such as phenytoin and corticosteroids, and recent consumption of calcium and vitamin D supplements (such as oral pills and injectable vitamin D3 and oral vitamin D2) during the past six months. Therefore, we enrolled 46 patients in our study and only one patient was excluded due to positive HBS antigen test.

\subsection{Laboratory Testing}

After recording anthropometric indices, blood pressure, baseline labs obtained including serum calcium (Ca), albumin (Alb), phosphorus (P), magnesium (Mg), Creatinine $(\mathrm{Cr})$, alkaline phosphatase (AlP), 25-OH-Vitamin D3 (25 (OH) D), intact parathyroid hormone (PTH), fasting blood sugar (FBS), Hemoglobin A1c (HbA1c), highdensity lipoprotein (HDL), low-density lipoprotein (LDL), triglyceride (TG), total cholesterol (t-Chol) and uric acid. All blood chemistries were obtained in Erfan Hospital clinical laboratory by standard methods.

$25(\mathrm{OH}) \mathrm{D}$ and PTH levels were measured using ELISA method (Immunodiagnostic Systems (IDS) Ltd, Boldon, England). The normal range of $25(\mathrm{OH}) \mathrm{D}$ is $30-150 \mathrm{ng} / \mathrm{mL}$. $25(\mathrm{OH}) \mathrm{D}$ concentration of 21 to $30 \mathrm{ng} / \mathrm{mL}$ is defined as vitamin D insufficiency. The normal range of PTH is $11-65.8$ $\mathrm{pg} / \mathrm{mL}(1.2-7 \mathrm{pmol} / \mathrm{L})$. Vitamin D deficiency is defined as vitamin $25(\mathrm{OH}) \mathrm{D} \leq 20 \mathrm{ng} / \mathrm{mL}$ and also hyperparathyroidism is defined as PTH $\geq 65.8 \mathrm{pg} / \mathrm{mL}(7 \mathrm{pmol} / \mathrm{L})$.

\subsection{Study Design}

Bariatric operations were performed by a single surgeon (K.T) at Erfan hospital, Tehran, Iran. During the laparoscopy operation, a wedge-shaped biopsy was taken from the left lateral segment of liver by the surgeon as a routine part of the procedure (21). All the biopsies were fixed and examined using Masson trichrome, silver reticulin and hematoxylin-eosin stain. A liver pathologist blinded to patients' clinical condition and laboratory data, reviewed the histology slides and reported "having
NASH" or "not-having NASH" using the following criteria for NASH definition:1) lobular necroinflammatory foci, 2) ballooning degeneration of hepatocytes with or without Mallory bodies, 3) perisinusoidal fibrosis (22). Diagnosis of metabolic syndrome (MetS) was made according to the international diabetes federation (IDF) definition (Central obesity plus any two of the following four factors: triglycerides $\geq 150 \mathrm{mg} / \mathrm{dL}$ or specific treatment for this lipid abnormality, HDL $<40 \mathrm{mg} / \mathrm{dL}$ in males, HDL $<$ $50 \mathrm{mg} / \mathrm{dL}$ in females or specific treatment for this lipid abnormality, systolic blood pressure (BP) $\geq 130 \mathrm{~mm} \mathrm{Hg}$ or diastolic BP $\geq 85 \mathrm{mmHg}$ or treatment of previously diagnosed hypertension, fasting plasma glucose $\geq 100 \mathrm{mg} /$ dL or previously diagnosed T2DM) (23). According to the latest studies in Iran, waist circumference cut-off point for the diagnosis of MetS and central obesity in Iranian adults is $90 \mathrm{~cm}$ for both genders (24).

This study was performed in accordance with the 1964 Declaration of Helsinki on medical protocol and ethics and the ethical committee of Tehran university of medical sciences approved the study. All patients gave their written informed consent prior to enrolling in the study.

\subsection{Statistical Analysis}

Data are expressed as mean \pm standard deviation unless otherwise indicated. The Kolmogorov-Smirnov test was applied to continuous variables to ensure a normal distribution of the variables. The significance of difference in continuous variables between groups was obtained by unpaired student's t-tests and $\chi^{2}$ test or Fisher's exact test was used for discontinuous variables. Multiple logistic regressions were performed to assess odds for NASH. We fitted three models (NASH predicting models) for predicting the presence of NASH. Model-1: serum PTH and its known related parameters including $\mathrm{Ca}, \mathrm{Mg}$, Creatinine, $25(\mathrm{OH}) \mathrm{D}$ and phosphorus considered as independent variables in a multivariate logistic regression that NASH (yes or no) was the dependent variable. Model-2: other possible confounding factors including age, gender, BMI, hypertension, current smoking, having diabetes mellitus type 2 (T2DM) and season of blood sampling were added to Model-1. Model-3: presence or absence of metabolic syndrome (MetS) was added to model-2 (15). Two-sided p value less than 0.05 considered as statistically significant for all tests. Data was analyzed using SPSS Statistics version 20 (SPSS Inc., Chicago, IL, USA).

\section{Results}

\subsection{Participants' Characteristics}

A total of forty-six obese subjects (females: 34 [74\%]; males: 12 [26\%]) with a mean age of $36.5 \pm 10.6$ years (ranged 18-54 years) and a mean BMI of $45 \pm 7.3 \mathrm{~kg} / \mathrm{m}^{2}$ included in this study. All patients were Iranian. Twenty $(43.5 \%)$ of the patients had NASH and the rest of 26 patients (56.5\%) did not fulfill the histological criteria 
Ghoghaei M et al.

for NASH. The prevalence of $25(\mathrm{OH})$ D deficiency $(\leq 20$ $\mathrm{ng} / \mathrm{mL}$ ) and hyperparathyroidism ( $\geq 65.8 \mathrm{pg} / \mathrm{mL}$ ) were 78.3\% (36 patients) and 32.6\% (15 patients), respectively. Thirty-two $(69.6 \%)$ of patients fulfilled the criteria of having metabolic syndrome and all patients had serum $\mathrm{Ca}$ and $\mathrm{P}$ in normal ranges.

\subsection{Characteristics According to Absence or Pres- ence of $\mathrm{NASH}$}

Biochemical and clinical characteristics of obese patients are shown in Table 1. Age, gender and BMI were not significantly different between groups. Although $25(\mathrm{OH})$ D levels were low in both groups, the mean did not differ significantly. Whereas, PTH, AST and ALT levels were significantly higher in the group with NASH (Pvalue $<0.001$ for PTH and
ALT, and $<0.01$ for AST). Frequency of MetS was significantly higher in the NASH group (90\% vs. 53.8\%) (Figure 1).

\subsection{PTH, $25(\mathrm{OH}) \mathrm{D}$ and Odds for Having NASH}

PTH was significantly correlated with $25(\mathrm{OH}) \mathrm{D}(\mathrm{r}=-0.308$, P value: 0.04). In unadjusted logistic model, PTH was the only variable in vitamin D endocrine system (Ca, P, $25(\mathrm{OH})$ D, PTH), which was significantly associated with NASH (odds ratio (OR): 1.0445, 95\% CI: 1.015 - 1.075, Nagelkerke's $\mathrm{R}^{2}=0.403, \chi^{2}=8.765$, P value: 0.003 ) in our morbidly obese patients (Figure 2). In NASH predicting logistic models introduced previously in methods, PTH was significantly associated with having NASH in all the three models (Table 2). In the second and third models, we can also see the age as the variable correlated with presence of NASH.

\begin{tabular}{|c|c|c|c|}
\hline & $\begin{array}{c}\text { Patients Without Non-Alcoholic } \\
\text { Steatohepatitis }(\mathbf{n}=\mathbf{2 6})\end{array}$ & $\begin{array}{c}\text { Patients With Non-Alcoholic } \\
\text { Steatohepatitis }(\mathbf{n}=\mathbf{2 0})\end{array}$ & PValue \\
\hline Gender & & & 0.227 \\
\hline Female & 81 & 65 & \\
\hline Male & 19 & 35 & \\
\hline Age, $y$ & $35.5 \pm 9.43$ & $39.0 \pm 10.1$ & 0.233 \\
\hline BMI, $\mathrm{kg} / \mathrm{m}^{2}$ & $44.3 \pm 5.82$ & $45.2 \pm 8.99$ & 0.704 \\
\hline AST, U/L & $20 \pm 9.68$ & $32.3 \pm 17.48$ & 0.009 \\
\hline ALT, U/L & $24.2 \pm 13.5$ & $44.2 \pm 24.8$ & 0.003 \\
\hline AST/ALT & $0.88 \pm 0.20$ & $0.76 \pm 0.16$ & 0.028 \\
\hline VitD $_{3}, \mathrm{ng} / \mathrm{mL}$ & $14.1 \pm 13.5$ & $8.71 \pm 5.58$ & 0.126 \\
\hline PTH, pg/mL & $40.4 \pm 19.7$ & $84.5 \pm 48.5$ & $<0.001$ \\
\hline Ca, mg/dL & $9.14 \pm 0.49$ & $9.20 \pm 0.42$ & 0.688 \\
\hline$P, \mathrm{mg} / \mathrm{dL}$ & $3.36 \pm 0.53$ & $3.20 \pm 0.56$ & 0.359 \\
\hline Mg, mg/dL & $2.12 \pm 0.21$ & $2.13 \pm 0.23$ & 0.886 \\
\hline Alb, g/dL & $4.36 \pm 0.52$ & $4.38 \pm 0.39$ & 0.887 \\
\hline FBS, mg/dL & $108.2 \pm 25.9$ & $109.8 \pm 25.2$ & 0.837 \\
\hline HbA1c, \% & $5.89 \pm 0.91$ & $6.22 \pm 1.08$ & 0.270 \\
\hline T-Chol, mg/dL & $192.4 \pm 36.4$ & $182.4 \pm 32.0$ & 0.344 \\
\hline LDL, mg/dL & $110.8 \pm 26.4$ & $107.0 \pm 23.0$ & 0.613 \\
\hline $\mathrm{TG}, \mathrm{mg} / \mathrm{dL}$ & $151.0 \pm 82.7$ & $158.7 \pm 58.0$ & 0.729 \\
\hline Alp, IU/L & $230.7 \pm 183.3$ & $193.1 \pm 63.5$ & 0.397 \\
\hline MetS, \% & 53.8 & 90 & 0.008 \\
\hline
\end{tabular}

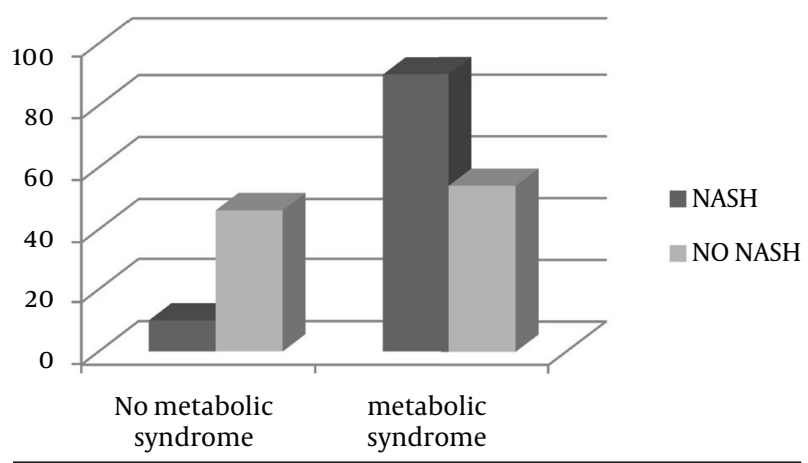

Figure 1. Percentage of Non-Alcoholic Steatohepatitis in Patients With and Without Metabolic Syndrome

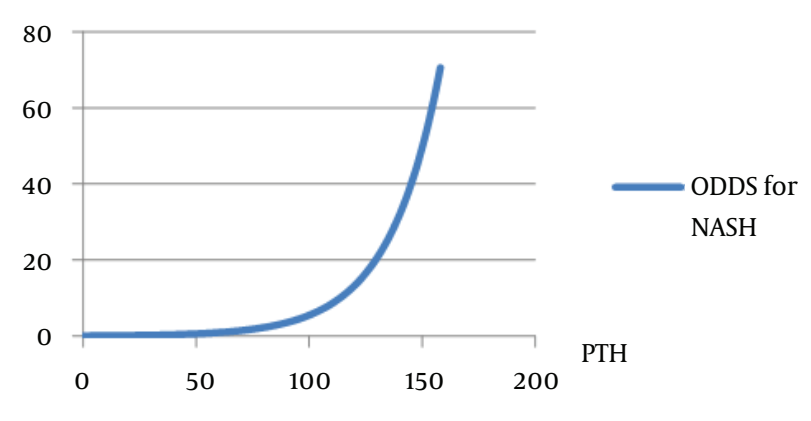

Figure 2. Odds for Non-Alcoholic Steatohepatitis 
Ghoghaei M et al.

\begin{tabular}{|c|c|c|c|c|c|c|}
\hline & \multicolumn{2}{|c|}{ Model-1 } & \multicolumn{2}{|c|}{ Model-2 } & \multicolumn{2}{|c|}{ Model-3 } \\
\hline & OR $(95 \% C I)$ & P Value & OR $(95 \% C I)$ & P Value & OR $(95 \% C I)$ & PValue \\
\hline PTH & $1.09(1.01-1.17)$ & 0.021 & $1.08(1.01-1.16)$ & 0.027 & $1.08(1.01-1.16)$ & 0.030 \\
\hline VitD $_{3}$ & $0.98(0.9-1.1)$ & 0.72 & $0.98(0.9-1.1)$ & 0.696 & $0.97(0.9-1.1)$ & 0.641 \\
\hline Age & - & - & $1.22(1.01-1.5)$ & 0.039 & $1.22(1.0-1.5)$ & 0.043 \\
\hline
\end{tabular}

a Abbreviations: CI, confidence interval; OR, odds ratio.

Table 3. Hyperparathyroidism and Non-Alcoholic Steatohepatitis a

\begin{tabular}{lcc}
\hline \multirow{2}{*}{ Patients } & \multicolumn{2}{c}{ Parathyroid Hormone } \\
\cline { 2 - 3 } & Normal & Elevated \\
\hline Patients without non-alcoholic steatohepatitis & 47.8 & 8.7 \\
Patients with non-alcoholic steatohepatitis & 19.6 & 23.9 \\
\hline
\end{tabular}

a Data are presented as \%.

To complete our work, we assessed quartiles of $25(\mathrm{OH})$ D and PTH as a dichotomous variable (hyperparathyroidism vs. normal PTH) instead of continuous variable and performed logistic regression for this new categorical independent variable; we again found no significant association between $25(\mathrm{OH})$ D quartiles and presence of NASH in obese patients, but the model of hyperparathyroidism and NASH showed significant association between these two new variables (Table 3) (odds ratio (OR): 6.722, Nagelkerke's $\mathrm{R}^{2}=0.220, \chi^{2}=7.298$, P value $\left.=0.007\right)$.

\subsection{Metabolic Syndrome and Presence of NASH}

In binary logistic regression model, in which metabolic syndrome (presence/absence) was introduced as an independent variable, metabolic syndrome was found to be a predictive factor for NASH (odds ratio: 7.714, 95\% CI: 1.48 - 40.2, Nagelkerke's $\mathrm{R}^{2}=0.205, \chi^{2}=5.88$, P value $=$ 0.015 ) (Figure 1), but after adjustment for PTH levels, this interaction did not remain significant anymore ( $P$ value $=0.112$, CI: $0.713-25.54$ ).

\subsection{AST, ALT and NASH}

Using logistic regression, when AST, ALT and AST: ALT ratio were considered separately as independent variables to predict NASH, a significant correlation was found between each of these variables with NASH (OR: 1.08 [95\%CI:1.015 - 1.146], 1.06 [95\%CI:1.015 - 1.102], 0.022 [95\%CI:0.001 - 0.854], respectively.

\subsection{Age at Liver Biopsy and NASH}

Age at liver biopsy and NASH did not show any correlation when it was considered the only independent variable, but after adjusting for other factors including PTH, sex, BMI, season of blood sampling, current smoking, MetS, Ca, Mg, $\mathrm{P}$ and vitD3, age showed its correlation with NASH (Table 2).

\section{Discussion}

The novel finding of this study was elevated serum PTH level as the predictive factor for NASH in morbidly obese patients seeking bariatric surgery, independent of other factors introduced previously.

\subsection{Prevalence of NASH}

In our study, $43.5 \%$ of forty-six morbidly obese patients, candidates for bariatric surgery met the criteria for NASH. Because of a number of factors such as silent nature of NAFLD and its important subgroup, NASH, reports about the prevalence of this disease in obese patients are quite variable. However, our findings are consistent with a study by Spaulding et al. (25) and Ong et al. (26), in which prevalence of NASH were $56 \%$ and $23.5 \%$ respectively, in morbidly obese patients. Although the difference was not significant, in our study this prevalence in men and women were $58.3 \%$ and $38.2 \%$ respectively, which is consistent with the study by Arun et al. (27), in which $60.3 \%$ of morbidly obese men and $30.9 \%$ of obese women were diagnosed with NASH.

\section{2. $\mathrm{PTH}, 25(\mathrm{OH}) \mathrm{D}$ and NASH}

This study demonstrated that PTH but not 25(OH) D was associated significantly with nonalcoholic steatohepatitis in morbidly obese patients. Although, several lines of evidence have already discussed the impact of vitamin D-endocrine system on obesity and its related comorbidities such as metabolic syndrome, there are very few published studies about the effect of this system on liver histopathology in obese subjects. Hjelmesaeth et al. (15) in a cohort study of 1017 morbidly obese patients reported that PTH is an independent predictor of metabolic syndrome in morbidly obese patients, whereas there is no association between MetS and $25(\mathrm{OH}) \mathrm{D}$. However, this 
finding was not confirmed by some other studies (28, 29). Targher et al. (30) found that NAFLD patients have a significant decrease in serum $25(\mathrm{OH})$ D levels, which is inversely associated with severity of liver histopathology (subjects were not selected from morbidly obese patients in this study).

Interestingly, despite PTH effects on bone and kidney, some hypotheses indicate that excess PTH may promote weight gain with increasing intra-adipocytes free calcium, thus blunting the lipolytic response to catecholamines (31).

\subsection{Liver Enzymes and NASH}

In our study, the mean level of both AST and ALT were higher in obese patients who met the histological criteria for NASH. Many previous studies also confirmed this finding and introduced elevated liver enzymes as the predictor of NASH $(10,14)$. Because liver enzymes might be in the normal range in patients with NASH, these two tests are not good predictors of this disease.

\subsection{Age and NASH}

Because of the potential association of age with other biological factors, we could not find the effect of age on presence of NASH at first, but after adjusting for other variables, an association found between age and NASH. Thus, as a morbidly obese patient gets older, the probability of progression to NASH would be increased. This finding is consistent with previous studies which introduced increasing age as a risk factor for $\operatorname{NASH}(10,13)$.

Recent consumption of calcium and vitamin D supplements can affect serum PTH and $25(\mathrm{OH})$ D levels, and it could be a limitation for determining the role of vitamin D endocrine system in predicting the probability of presence of NASH. We excluded patients with recent consumption of calcium or vitamin D supplements from the study to overcome this limitation.

In conclusion, the present study found elevated serum liver enzymes, high serum PTH levels and older age as predictors of NASH in morbidly obese patients seeking obesity surgical treatments. If studies with large sample sizes from different ethnic groups confirm our findings, serum PTH level may help clinicians to screen patients with NASH. However, liver biopsy is still the gold standard for detecting NASH.

\section{Acknowledgements}

Thanks to Dr. Soudeh Taghdiri and B.P.Resnick for reviewing the English language.

\section{Authors' Contributions}

Morteza Ghoghaei and Karamollah Toolabi conceived the study and contributed to recruitment of patients. Elias Khajeh, Foad Taghdiri, Sepideh Hosseini Shirvani, and Shadi Zarei were involved in recruitment of patients and collecting data. Farid Azmoudeh Ardalan did the pathological examinations and reports. Elias Khajeh and Sepideh Hosseini Shirvani drafted the manuscript, Foad Taghdiri, Shadi Zarei, and Mojtaba Sedaghat conducted statistical analysis and contributed to manuscript drafting. Elias Khajeh and Morteza Ghoghaei edited and finalized the manuscript. All authors read and approved the submitted manuscript.

\section{Financial Disclosure}

This study was funded by Tehran University of Medical Sciences.

\section{References}

1. Ezquerra EA, Vázquez JMC, Barrero AA. Obesity, Metabolic Syndrome, and Diabetes: Cardiovascular Implications and Therapy. Rev Esp Cardiol. 2008;61(7):752-64.

2. Low S, Chin MC, Deurenberg-Yap M. Review on epidemic of obesity. Ann Acad Med Singapore. 2009;38(1):57-9.

3. Nahleh Z, Bhatti NS, Mal M. How to reduce your cancer risk: mechanisms and myths. Int J Gen Med. 2011;4:277-87.

4. Machado M, Marques-Vidal P, Cortez-Pinto H. Hepatic histology in obese patients undergoing bariatric surgery. J Hepatol. 2006;45(4):600-6.

5. Gholam PM, Kotler DP, Flancbaum LJ. Liver pathology in morbidly obese patients undergoing Roux-en-Y gastric bypass surgery. Obes Surg. 2002;12(1):49-51.

6. Del Gaudio A, Boschi L, Del Gaudio GA, Mastrangelo L, Munari D. Liver damage in obese patients. Obes Surg. 2002;12(6):802-4.

7. Caldwell SH, Oelsner DH, Iezzoni JC, Hespenheide EE, Battle EH, Driscoll CJ. Cryptogenic cirrhosis: clinical characterization and risk factors for underlying disease. Hepatology. 1999;29(3):664-9.

8. Hashimoto E, Tokushige K. Prevalence, gender, ethnic variations, and prognosis of NASH.J Gastroenterol. 2011;46 Suppl 1:63-9.

9. Harnois F, Msika S, Sabate JM, Mechler C, Jouet P, Barge J, et al. Prevalence and predictive factors of non-alcoholic steatohepatitis (NASH) in morbidly obese patients undergoing bariatric surgery. Obes Surg. 2006;16(2):183-8.

10. Crespo J, Fernandez-Gil P, Hernandez-Guerra M, Cayon A, Mayorga M, Dominguez-Diez A, et al. Are there predictive factors of severe liver fibrosis in morbidly obese patients with non-alcoholic steatohepatitis? Obes Surg. 2001;11(3):254-7.

11. Ratziu V, Giral P, Charlotte F, Bruckert E, Thibault V, Theodorou I, et al. Liver fibrosis in overweight patients. Gastroenterol. 2000;118(6):1117-23.

12. Dixon JB, Bhathal PS, O'Brien PE. Nonalcoholic fatty liver disease: predictors of nonalcoholic steatohepatitis and liver fibrosis in the severely obese. Gastroenterology. 2001;121(1):91-100.

13. Angulo P, Keach JC, Batts KP, Lindor KD. Independent predictors of liver fibrosis in patients with nonalcoholic steatohepatitis. Hepatology. 1999;30(6):1356-62.

14. Amarapurka DN, Amarapurkar AD, Patel ND, Agal S, Baigal R, Gupte P, et al. Nonalcoholic steatohepatitis (NASH) with diabetes: predictors of liver fibrosis. Ann Hepatol. 2006;5(1):30-3.

15. Hjelmesaeth J, Hofso D, Aasheim ET, Jenssen T, Moan J, Hager H, et al. Parathyroid hormone, but not vitamin D, is associated with the metabolic syndrome in morbidly obese women and men: a cross-sectional study. Cardiovasc Diabetol. 2009;8:7.

16. Wortsman J, Matsuoka LY, Chen TC, Lu Z, Holick MF. Decreased bioavailability of vitamin D in obesity. Am J Clin Nutr. 2000;72(3):690-3.

17. Moreiro J, Ruiz O, Perez G, Salinas R, Urgeles JR, Riesco M, et al. Parathyroid hormone and bone marker levels in patients with morbid obesity before and after biliopancreatic diversion. Obes Surg. 2007;17(3):348-54.

18. Goldner WS, Stoner JA, Thompson J, Taylor K, Larson L, Erickson $\mathrm{J}$, et al. Prevalence of vitamin D insufficiency and deficiency in morbidly obese patients: a comparison with non-obese controls. Obes Surg. 2008;18(2):145-50. 


\section{Ghoghaei M et al.}

19. Toolabi K, Arefanian S, Golzarand M, Arefanian H. Effects of laparoscopic Roux-en-Y gastric bypass (LRYGB) on weight loss and biomarker parameters in morbidly obese patients: a 12-month follow-up. Obes Surg. 2011;21(12):1834-42.

20. Hubbard VS, Hall WH. Gastrointestinal Surgery for Severe Obesity. Obes Surg. 1991;1(3):257-65.

21. Shalhub S, Parsee A, Gallagher SF, Haines KL, Willkomm C, Brantley SG, et al. The importance of routine liver biopsy in diagnosing nonalcoholic steatohepatitis in bariatric patients. Obes Surg. 2004;14(1):54-9.

22. Lee RG. Nonalcoholic steatohepatitis: tightening the morphological screws on a hepatic rambler. Hepatology.1995;21(6):1742-3.

23. Alberti KG, Zimmet P, Shaw J, I. D. F. Epidemiology Task Force Consensus Group . The metabolic syndrome--a new worldwide definition. Lancet. 2005;366(9491):1059-62.

24. Esteghamati A, Ashraf H, Rashidi A, Meysamie A. Waist circumference cut-off points for the diagnosis of metabolic syndrome in Iranian adults. Diabetes Res Clin Pract. 2008;82(1):104-7.

25. Spaulding L, Trainer T, Janiec D. Prevalence of non-alcoholic steatohepatitis in morbidly obese subjects undergoing gastric bypass. Obes Surg. 2003;13(3):347-9.

26. Ong JP, Elariny H, Collantes R, Younoszai A, Chandhoke V,
Reines HD, et al. Predictors of nonalcoholic steatohepatitis and advanced fibrosis in morbidly obese patients. Obes Surg. 2005;15(3):310-5.

27. Arun J, Clements RH, Lazenby AJ, Leeth RR, Abrams GA. The prevalence of nonalcoholic steatohepatitis is greater in morbidly obese men compared to women. Obes Surg. 2006;16(10):1351-8.

28. Botella-Carretero JI, Alvarez-Blasco F, Villafruela JJ, Balsa JA Vazquez C, Escobar-Morreale HF. Vitamin D deficiency is associated with the metabolic syndrome in morbid obesity. Clin Nutr 2007;26(5):573-80.

29. Kayaniyil S, Vieth R, Harris SB, Retnakaran R, Knight JA, Gerstein $\mathrm{HC}$, et al. Association of $25(\mathrm{OH}) \mathrm{D}$ and PTH with metabolic syndrome and its traditional and nontraditional components. J Clin Endocrinol Metab. 2011;96(1):168-75.

30. Targher G, Bertolini L, Scala L, Cigolini M, Zenari L, Falezza G, et al. Associations between serum 25-hydroxyvitamin D3 concentrations and liver histology in patients with non-alcoholic fatty liver disease. Nutr Metab Cardiovasc Dis. 2007;17(7):517-24.

31. McCarty MF, Thomas CA. PTH excess may promote weight gain by impeding catecholamine-induced lipolysis-implications for the impact of calcium, vitamin D, and alcohol on body weight. Med Hypotheses. 2003;61(5-6):535-42. 